\title{
Bericht über die
}

\section{Jahresversammlung der Deutschen Ornithologischen Gesellschaft in Hamburg und auf Helgoland}

\author{
vom 6. bis 9. Juni 1905 .
}

Die Tagung fand gemeinsam mit der Jahresversammlung des Deutschen Vereines zum Schutze der Vogelwelt statt.

Es waren von den Mitgliedern unserer Gesellschaft folgende Herren zugegen:

Reichenow, Paeske, Grunack, Matschie, Heinroth und von Lucanus aus Berlin, R. Blasius (Braunschweig), Kollibay (Neisse), Hennicke (Gera), Kuschel (Guhrau), Kräp elin (Hamburg), Talsky (Olmütz), Hanke (Kentschkau).

Von den Mitgliedern des Deutschen Vereines zum Schutze der Vogelwelt, soweit sie nicht Mitglieder unserer Gesellschaft sind, hatten sich folgende Herren beteiligt:

Kensing (Breslau), Jahn (Hohenleuben), Gechter (Neuwerk), Freiherr von Wangenheim (Röcknitz), von Wange lin (Merseburg), Krohn (Hamburg), Li ind n er (Osterwieck), Lind ner (Wettaburg), Taschenberg (Halle a/S.), Schultze (Dresden).

Als Gäste waren anwesend die Herren: Martens, Graemer, Kr ieger, Lohff, Schultz (Hamburg), Professor Dr. Ehrenbaum, Professor Dr. Hartlaub, Dr. Immermann, Dr. Bolau, Dr. Strodtmann (Helgoland) und die Damen: Frau Heinroth, Frau von Lucanus (Berlin), Frau Hartlaub, Frau Ehrenbaum, Frau Immermann und Frau Bolau (Helgoland).

\section{Vorversammlung im Zoologischen Garten zu Hamburg.}

Am Dienstag, den 6. Juni 1905, Abends 7 Uhr.

In zwangloser Weise fand im Zoologischen Garten die Begrüssung der aus vielen Teilen Deutschlands herbeigeeilten Vogelkenner statt. Unser Mitglied, Herr Dr. Bolau, der Direktor des Hamburger Zoologischen Gartens, hatte leider auf die Beteiligung an der Jahresversammlung verzichten müssen, weil er durch eine bei einem Eisenbahnunfall entstandene Verletzung an das Zimmer gefesselt war. Ihm verdanken die Mitglieder freien Eintritt in den Garten während der ganzen Dauer der Versammlung.

In einer kurzen Besprechung, die in einem der Restaurationssäle stattfand, wurde der Arbeitsplan für die folgenden Tage festgestellt.

\section{Fahrt nach Helgoland. Einweihung der Gedenktafel für Gätke. Wissenschaftliche Sitzung.}

Am Mittwoch, den 7. Juni 1905.

Morgens um 8 Uhr lichtete die „Cobra" die Anker, sie brachte bei sehr schönem Wetter die Mitglieder in 7 Stunden 
nach Helgoland. Die Fahrt bot wiederholt Gelegenheit zu ornithologischen Beobachtungen.

Im Kurhause wurde zunächst ein gemeinsames Mittagessen eingenommen.

Um 6 Uhr fand alsdann die Einweihung einer Gedenktafel für den bekannten Ornithologen Heinrich Gätke an dem von ihm 1837-1897 bewohnten Hause statt.

Herr Blasius schilderte in Gätke's Atelier mit begeisterten Worten die Verdienste des unermüdlichen Beobachters, sprach seine Freude darüber aus, dass Gätke's Lebenswerk schon in der „Vogelwarte Helgoland" ein von allen Ornithologen mit lebhafter Befriedigung begrüsstes Denkmal gesetzt sei; die von ihm in vielen Jahren mit mühsamer Arbeit zusammengebrachte Sammlung stehe in den Räumen der zoologischen Station allen Vogelfreunden zur Benutzung offen. Jetzt gehe ein Wunsch der deutschen Ornithologen in Erfüllung, das Haus, in dem Gätke Jahrzehnte lang wohnte und wirkte, mit einem dauernden Andenken für die Nachwelt zu versehen. Auf Veranlassung des Deutschen Vereines zum Schutze der Vogelwelt und der Deutschen Ornithologischen Gesellschaft sind die Mittel zur Anfertiguug einer Erztafel bereit gestellt worden.

Nunmehr fiel die Hülle von der Gedenktafel.

Nachdem der Garten des Hauses, in dem Gätke viele seiner Erfahrungen gemacht hatte, besichtigt war, dankte Gätke's Sohn, Herr Ludwig Gätke, mit herzlichen Worten für die Ehrung seines Vaters.

Nach einem Rundgange um die Insel, der auch Gelegenheit geboten hatte, die Bewohner des Lummenfelsens aus nächster Nähe zu beobachten, vereinigten sich die Mitglieder der Jahresversammlung um 9 Uhr zu einer wissenschaftlichen Sitzung im Kurhause.

Herr von Wangelin übernahm den Vorsitz.

Herr Blasius begrüsste die Anwesenden und teilte mit, dass die Herren Hans Freiherr von Berlepsch, Regierungsrat Professor Dr. Rörig und Dr. med. Schnee zu ihrem grössten Bedauern sich an dieser Versammlung nicht beteiligen könnten und demnach die von ihnen angekündigten Vorträge zu halten nicht im Stande seien.

Herzliche Grüsse hatten teils telegraphisch teils anderweitig gesandt die Herren: W. Blasius (Braunschweig), Thienemann (Rossitten), Arends und Bachmann (Juist), Reiser (Sarajewo), Rohweder (Husum).

Herr Blasius hielt nunmehr den einzigen noch angemeldeten Vortrag: "Die hohe Tatra und ihre Vogelwelt." Der Redner gab u. a. einige Mitteilungen über einzelne in der Sammlung von Poprad aufbewahrte Vögel, berichtete über ein Nest von Turdus torquatus alpestris, das in einem ausgehöhlten Baumstamm stand, und über die ornithologischen Sammlungen in den Museen 
von Zakopane, Poprad und Felka und die Sammlung des Dr. Greisiger in Szepes-Bela.

Herr von Wangelin dankte dem Vortragenden und eröffnete die Besprechung. An ihr nahmen Teil die Herren: von Wangelin, Kollibay, Talsky, Krohn und Blasius.

Herr von Wangelin schlug hierauf vor, dass die Mitglieder des Deutschen Vereines zum Schutze der Vogelwelt am Donnerstag Abend im Hôtel Graf Moltke in Hamburg zu einer geschäftlichen Sitzung sich vereinigen sollten.

Nach einigen Mitteilungen über die für den nächsten Tag festgesetzten Unternehmungen wurde die Sitzung um $1 / 211$ Uhr geschlossen. Der spätere Abend bot bei ernsten und heiteren Gesprächen Gelegenheit, alte Freundschaftsbeziehungen zu festigen und neue anzuknüpfen.

\section{Bootfahrt zu dem Lummenfelsen. Besichtigung des Museums und Aquariums. Fahrt nach Hamburg.}

\section{Am Donnerstag, den 8. Juni 1905.}

Um 7 Uhr wurde eine Fahrt zu dem Lummenfelsen unternommen. Die See war etwas bewegt. Die Flutverhältnisse gestatteten nicht, nahe genug heranzufahren; trotzdem wird den Teilnehmern an dieser schönen Fahrt das Gesehene in frischer Erinnerung bleiben. Von den stark besetzten Felskanten erhoben sich immer wieder Schwärme der Lummen, strichen schnellen Fluges zum Meere hinaus und kehrten dann in weitem Bogen zurück. Treppenstufen gleich hoben sich die Ketten weisser Vögel von dem roten Felsen ab, an manchen Stellen in 20 Reihen übereinander.

Nach der Rückkehr fanden sich alle Teilnehmer in dem Nordsee-Museum wieder zusammen. Die Herren Professor Dr. Hartlaub und Professor Dr. Ehrenbaum erklärten die bemerkenswertesten Ausstellungsgegenstände und wurden nicht müde, die immer wieder neu an sie gerichteten Fragen in liebenswürdigster Weise zu beantworten. Hier ist wohl überflüssig, die Vorzüge des Helgoländer Museums besonders hervorzuheben. Jeder Ornithologe weiss, dass Gätke's Vogelsammlung dort aufgestellt ist. Vielleicht darf aber daran erinnert werden, dass die meisten Vögel in mustergiltiger Weise umgestopft, viele Neuigkeiten hinzugekommen und namentlich auf die Aufstellung biologischer Gruppen grosser Wert gelegt worden ist.

Aus dem Nordsee-Museum ging der Weg zur Zoologischen Station und zum neu eröffneten Aquarium, einer Muster-Anstalt, die den Vergleich mit den berühmten Schwesterstätten in Brighton und Neapel nicht zu scheuen braucht.

Nur ungern trennte man sich von allen den wunderbaren Eindrücken, die hier in den Seewasserbecken die ganze Pracht der Meerestierwelt den Augen darbot. 
Die Zeit verfloss zu schnell. In aller Eile konnten die Ornithologen noch ein Frühstück einnehmen, dann galt es, die Boote zu besteigen, um die "Cobra“ wieder zu erreichen.

Mit herzlichem Abschiede von den Helgoländer Zoologen, deren freundlichem Wirken diese Jahresversammlung einen beträchtlichen Teil des Gelingens verdankt, verliessen wir den Boden der roten Insel. Die See ging nicht gerade hoch, zeigte doch aber die Neigung, ihre Wellenkämme in lebhafter Bewegung zu wiegen. So sah es denn auf dem Schiffe bald ziemlich trübe aus; viele der Reisenden verloren ihr blühendes Aussehen, gar mancher musste den keineswegs beneidenswerten Zustand, den man Seekrankheit nennt, kennen lernen; aber merkwürdigerweise hielten sich alle Mitglieder der Jahresversammlung, auch die Damen, sehr gut. Unterwegs bot sich uns wiederholt der Genuss, grosse Kriegsschiffe in nächster Nähe zu beobachten. Der Abend wurde von den meisten Mitgliedern unserer Gesellschaft dazu benutzt, um mit den Herren des Deutschen Vereins zum Schutze der Vogelwelt noch einige Stunden zu plaudern; einige nahmen dagegen Gelegenheit zu einem Zusammensein mit den Hamburger Zoologen.

\section{Besichtigung des Naturhistorischen Museums in Hamburg. Hafenrundfahrt. Frühstiick. Wissenschaftliche Sitzung. Besichtigung des Zoologischen Gartens. Festessen. Schluss der Jahresversammlung.}

Am Freitag, den 9. Juni 1905.

Der Rundgang durch das Naturhistorische Museum fand in mehreren Gruppen statt. Die Herren Professor Dr. Kräpelin, Professor Dr. Pfeffer und Martens hatten die Führung freundlichst übernommen. Leider war die Zeit viel zu kurz, um die herrlichen Sammlungen eingehender besichtigen zu können. Nach einem flüchtigen Blick musste man weiter, aber die Überzeugung prägte sich doch jedem ein, dass das Hamburger Museum in der gesamten Anordnung und insbesondere in der Auswahl der zur Belehrung weiterer Kreise dienenden Darstellungen vorzüglich geleitet und verwaltet ist.

Um 12 Uhr erfolgte eine Besichtigung des Hafens.

Nach einem Frühstück im St. Pauli-Fährhause wurde im Vortragssaale des Naturhistorischen Museums die zweite wissenschaftliche Sitzung unter dem Vorsitze des Herrn Blasius um $1 \frac{1}{2}$ Uhr eröffnet.

Herr Matschie sprach zunächst über die Vogelwelt der mikronesischen Inseln. Er erinnerte an seine im Journal f. Ornithologie, Jahrg. 1901 p. 109-114 gemachten Mitteilungen, in denen er das Vorhandensein mehrerer zoogeographischer Gebiete nachzuweisen versucht hatte und teilte mit, dass neuerdings in dem Deutschen Kolonialblatt, 1905, XVI p. 328, Herr 
Bezirksamtmann Sennft eine Karte der Sprachgebiete Mikronesiens veröffentlicht habe. deren Grenzen in überraschender Weise mit denjenigen der von ihm besprochenen Tiergebiete übereinstimmen.

Hierauf sprach Herr Blasius über seine ornithologischen Erfahrungen auf der iberischen Halbinsel. Er schilderte besonders eine Excursion nach der Albufera bei Valencia, die er unter der Führung des Professor Bosca unternommen, eine Besichtigung des Felsens von Gibraltar und eine Tour in die Marismen des Guadalquiwir bei Sevilla. Die naturhistorischen Museen in Valencia, Granada, Salamanca, Madrid und Lissabon wurden einer kurzen Besprechung unterworfen.

Herr Matschi e gab hierzu einige Ergänzungen.

Dann wurde die Sitzung mit einem herzlichen Danke an Herrn Professor Kräpelin geschlossen.

Vom Museum begaben sich die Mitglieder zum Zoologischen Garten. Während des Rundganges bot sich die erwünschte Gelegenheit, über viele Seltenheiten die Ansichten auszutauschen.

Ein Festessen im Restaurationsgebäude des Gartens vereinigte die Teilnehmer zum letzten Male. Speisen und Getränke waren vorzüglich, einige Reden trugen dazu bei, die Stimmung zur Fröhlichkeit zu erhöhen und als der Abend hereingebrochen war, behauptete allgemeiner Frohsinn das Feld. Nur ungern trennte man sich in später Stunde. Die Hamburger Jahresversammlung hatte einen so befriedigenden Verlauf genommen, dass jeder Teilnehmer mit Freude sich ihrer erinnern wird.

Mehrere photographische Aufnahmen waren gemacht worden. Sie werden den Teilnehmern ein wertvolles Andenken an diese schönen Tage bewahren helfen.

P. Matschie.

\section{Dem Herausgeber zugesandte: Schriften.}

The Annals of Scottish Natural History. A Quarterly Magazine. Edinburgh. No. 55. 1905.

The Auk. A Quarterly Journal of Ornithology. Vol. XXII. No. 3. 1905.

Bird Notes and News. Circular Letter issued periodically by the Royal Society for the Protection of Birds. No. 10. London 1905.

Boletim do Museu Goeldi (Museu Paraense) de Historia Natural e ethnographia. Vol. IV. No. 1-3. Para 1904.

Bulletin de la Société Philomathique de Paris. 9 sér. T. VII. No. 2-3. 1905.

The Condor. A Magazine of Western Ornithology. Vol. VII. No. 3-5. 1905. 


\section{$2 \mathrm{BHL}$ Biodiversity Heritage Library}

1905. "Bericht über die 55. Jahresversammlung der Deutschen

Ornithologischen Gesellschaft in Hamburg und auf Helgoland." Journal fu

r Ornithologie 53, 757-761. https://doi.org/10.1007/bf02125170.

View This Item Online: https://www.biodiversitylibrary.org/item/107488

DOI: https://doi.org/10.1007/bf02125170

Permalink: https://www.biodiversitylibrary.org/partpdf/142382

\section{Holding Institution}

Smithsonian Libraries

\section{Sponsored by}

Biodiversity Heritage Library

\section{Copyright \& Reuse}

Copyright Status: Public domain. The BHL considers that this work is no longer under copyright protection.

This document was created from content at the Biodiversity Heritage Library, the world's largest open access digital library for biodiversity literature and archives. Visit BHL at https://www.biodiversitylibrary.org. 